\title{
A
}

Acta HealthMedica

Acta HealthMedica (ISSN: 2414-6528)

http://www.ActaHealthMedica.com

Volume 2, Issue 2, April-June 2017, Pages: 199, DOI: http://dx.doi.org/10.19082/ah199

\section{PATIENTS REGISTRIES WORLDWIDE}

\author{
Hossein Siabanil, Soraya Siabani 2
}

1: MD - Coordinator of ACS registry, Kermanshah University of Medical Sciences (KUMS)

2: MD, PhD - Assistant Professor at Department of Health Promotion, Public Health School, Kermanshah

\section{Correspondence:}

Kermanshah University of Medical Sciences (KUMS) \& Registered External Supervisor at the University technology Sydney (UTS), NSW, Australia

\section{TYPE OF ARTICLE: CONFERENCE ABSTRACT}

\begin{abstract}
Introduction: A disease/patients' registry, is a collection of secondary data related to those with particular condition/disease/procedure. Patients registries not only provide health providers and patients with reminders to check certain examinations in order to achieve certain quality goals, but their main role is associated to health research either as a database for clinical studies in a particular disease or (preferably) as a comprehensive cohort study by itself. The aim of this study is to summarize information published electronically about patients' registries.

Methods: This short report is resulted from reviewing the electronic databases and websites aiming to collect valid and comprehensive information in regard to the background and frequency of patients' registries worldwide. In addition, using the terms "patients' registries vs cohort studies", "patients' registry vs observational study", "patients' registry vs medical records" the variety, advantages and disadvantages of patients' registries were searched for.

Results: The results showed that a long list of disease registries from Europe, United States of America (USA) and Asian countries were detailed in publication. Formal disease registries have been given more attention from 1960, in which cancer registries were highlighted, however the absolute origin for patients' registries is not realized precisely. More than 5o patients' registries from USA only, have been introduced. The obtained information was mostly about registries at national or international levels and hence they were not including many local registries within the limited geographic area. Rare conditions (e.g., Usher Syndrome Registry) and chronic diseases (e.g., Alzheimer's Prevention Registry) are the most common cases that have systematically been registered.

Conclusion: A wide range of disease registration methods from sophisticated and complex databases to simple spreadsheets accessible for only a small group of physicians working in local health care centers, have been developed worldwide, however the more developed the country, the more diseases registry organizations there are. In other words, disease registries require human resources as well as fundamental constructions and amenities. Considering the advantages and benefits of disease registries, we recommend developing disease registries widely in our country, using the prepared and developed software and questionnaires from other countries.
\end{abstract}

KEYWORDS: Disease registry, patients' registry, registry researches, epidemiological studies

\section{Abstracts of First National Congress of Medical Informatics, Mashhad, Iran, February 2017}

(C) 2017 The Authors. This is an open access article under the terms of the Creative Commons Attribution-NonCommercialNoDerivs License, which permits use and distribution in any medium, provided the original work is properly cited, the use is non-commercial and no modifications or adaptations are made. 